\title{
Glycosylation and an amino acid insertion in the head of hemagglutinin independently affect the antigenic properties of H5N1 avian influenza viruses
}

\author{
Chunyang $\mathrm{Gu}^{1}$, Xianying Zeng ${ }^{1}$, Yangming Song ${ }^{1}$, Yanbing $\mathrm{Li}^{1}$, Liling $\mathrm{Liu}^{1}$, \\ Yoshihiro Kawaoka ${ }^{2}$, Dongming Zhao ${ }^{1 *} \&$ Hualan Chen ${ }^{1 *}$ \\ ${ }^{1}$ State Key Laboratory of Veterinary Biotechnology, Harbin Veterinary Research Institute, Chinese Academy of Agricultural Sciences, \\ Harbin 150069, China; \\ ${ }^{2}$ Division of Virology, Department of Microbiology and Immunology, Institute of Medical Science, University of Tokyo, Tokyo, Japan
}

Received November 18, 2018; accepted November 27, 2018; published online November 30, 2018

\begin{abstract}
Antigenic drift forces us to frequently update influenza vaccines; however, the genetic basis for antigenic variation remains largely unknown. In this study, we used clade $7.2 \mathrm{H} 5$ viruses as models to explore the molecular determinants of influenza virus antigenic variation. We generated eight monoclonal antibodies (MAbs) targeted to the hemagglutinin (HA) protein of the index virus $\mathrm{A} /$ chicken/Shanxi/2/2006 and found that two representative antigenically drifted clade 7.2 viruses did not react with six of the eight MAbs. The E131N mutation and insertion of leucine at position 134 in the HA protein of the antigenically drifted strains eliminated the reactivity of the virus with the MAbs. We also found that the amino acid N131 in the H5 HA protein is glycosylated. Our results provide experimental evidence that glycosylation and an amino acid insertion or deletion in HA influence antigenic variation.
\end{abstract}

Influenza virus, H5N1, antigenic variation, genetic basis

Citation: Gu, C., Zeng, X., Song, Y., Li, Y., Liu, L., Kawaoka, Y., Zhao, D., and Chen, H. (2019). Glycosylation and an amino acid insertion in the head of hemagglutinin independently affect the antigenic properties of H5N1 avian influenza viruses. Sci China Life Sci 62, 76-83. https://doi.org/10.1007/ s11427-018-9439-0

\section{INTRODUCTION}

Influenza viruses are widely circulating in nature; they can infect humans, various other mammals, and avian species. The natural hosts of influenza viruses are wild birds and waterfowl, from which influenza viruses bearing different subtypes of hemagglutinin (HA) (H1-H16) and neuraminidase (NA) (N1-N9) have been detected. H1N1, H2N2, and $\mathrm{H} 3 \mathrm{~N} 2$ viruses have caused four influenza pandemics since 1918, and H1N1 and H3N2 viruses continue to cocirculate in humans globally. In recent years, several sub-

*Corresponding authors (Dongming Zhao, email: zhaodongming@caas.cn; Hualan Chen, email: chenhualan@caas.cn) types of avian influenza virus, including H5N1, H7N7, H7N9, and H9N2, have been reported to infect humans and exhibit pandemic potential (Fouchier et al., 2004; Herfst et al., 2012; Imai et al., 2012; Kimble et al., 2011; Li et al., 2014b; Sorrell et al., 2009; World Health Organization, 2014; Zhang et al., 2013a; Zhang et al., 2013b). The H5N1 viruses have caused disease outbreaks in poultry and wild birds in more than 60 countries across three continents since 2003 (Li et al., 2014a; Swayne, 2012). Over 800 human infections have been reported in 16 countries with a mortality rate of nearly $60 \%$ (World Health Organization, 2018b). The H7N9 viruses caused more than 1,560 human infections between March 2013 and February 2018 with a mortality rate of nearly 40\% (World Health Organization, 2018a). After an 
H7N9 vaccine was introduced in poultry in September 2017, the prevalence of the H7N9 virus in poultry was prevented and subsequently human infections with H7N9 virus were also successfully eliminated (Shi et al., 2018; Wei and Cui, 2018; Zeng et al., 2018).

Vaccination is an important strategy to protect humans or animals from influenza virus infection. The HA protein of influenza virus is a surface glycoprotein and is the main target of neutralizing antibodies. Influenza viruses can mutate during their replication. When mutations in the HA protein alter the antigenicity of the viruses, antigenic drift strains will escape from the host's preexisting immunity and cause a new influenza epidemic. In such a situation, the current vaccine must be updated. Currently, vaccine seed viruses are updated only after antigenically drifted strains are prevalent in nature. Identification of genetic determinants for antigenic variation would therefore provide important insights for screening and early detection of antigenically drifted viruses, which in turn would be invaluable for vaccine preparation.

H5N1 viruses have evolved into 10 phylogenetic clades $(0-$ 9) based on the genetic diversity of their HA genes (WHOOIE-FAO H5 evolution working group, 2008). The clade 7.2 H5N1 viruses were initially detected in chickens in Shanxi province in China in 2006 and subsequently spread to chickens in several other northern provinces (Li et al., 2010; Liu et al., 2016). The viruses reassorted with other influenza viruses and formed different genotypes of H5N1 and H5N2 viruses (Liu et al., 2016). To control the clade 7.2 viruses, an inactivated vaccine containing the modified $\mathrm{HA}$ gene of the $\mathrm{CK} / \mathrm{SX} / 2 / 06$ virus (the index strain of clade 7.2), designated Re-4, was developed and had been used to control clade 7.2 virus infection of chickens in China since 2006 (Li et al., 2014a). However, viruses isolated between 2011 and 2014 showed antigenic drift from $\mathrm{CK} / \mathrm{SX} / 2 / 06$, and a new vaccine (termed Re-7) with a seed virus that bears the modified HA of $\mathrm{A} /$ chicken/Liaoning/S4092/2011 (CK/LN/S4092/11) was devel- oped and has been used in place of Re-4 since 2014 to control the clade 7.2 viruses (Liu et al., 2016). In this study, we used the clade $7.2 \mathrm{H} 5$ viruses as models to explore the genetic changes that affect the antigenic properties of influenza viruses.

\section{RESULTS}

\section{The clade $7.2 \mathrm{H5}$ viruses form three distinct antigenic groups}

To fully understand the antigenic relationships of the clade 7.2 H5 viruses, we selected nine viruses that were isolated between 2006 and 2013 and investigated their cross-reactivity with chicken antisera induced by an HA DNA vaccine of each of seven viruses by using the hemagglutinin inhibition (HI) test (Table 1). These antigenic data were also analyzed quantitatively by using the methods described by Smith et al. (Smith et al., 2004). The nine viruses formed three different antigenic clusters: cluster I, which contain the index isolate $\mathrm{A} /$ chicken/Shanxi/2/2006(H5N1) (CK/SX/2/ 06); cluster II, which includes A/chicken/Jiangsu/18/2008 (H5N1) (CK/JS/18/08) and A/chicken/Shandong/A-8/2011 (H5N1) (CK/SD/A-8/11); and cluster III, which contains the other six strains, namely A/chicken/Hebei/A/2012(H5N2) (CK/HeB/A/12), A/chicken/Liaoning/S4068/2011(H5N1) (CK/LN/S4068/11), A/chicken/Liaoning/S4092/2011(H5N1) (CK/LN/S4092/11), A/chicken/Ningxia/2/2012(H5N1) (CK/ NX/2/12), A/chicken/Gansu/6/2012(H5N1) (CK/GS/6/12), and $\mathrm{A} /$ chicken/Hebei/3/2013(H5N2) (CK/HeB/3/13) (Figure 1). These results demonstrate that the $\mathrm{CK} / \mathrm{JS} / 18 / 08$-like viruses and the $\mathrm{CK} / \mathrm{HeB} / \mathrm{A} / 12$-like viruses are antigenically drifted from the $\mathrm{CK} / \mathrm{SX} / 2 / 06$ virus and from each other.

\section{The $\mathrm{CK} / \mathrm{JS} / 18 / 08$ and $\mathrm{CK} / \mathrm{HeB} / \mathrm{A} / 12$ viruses do not react with monoclonal antibodies (MAbs) generated against the $\mathrm{CK} / \mathrm{SX} / 2 / 06$ virus}

An amino acid mutation in the epitope of HA may alter the

Table 1 Antigenic analysis of clade 7.2 H5 subtype avian influenza viruses

\begin{tabular}{|c|c|c|c|c|c|c|c|}
\hline \multirow{2}{*}{ Virus } & \multicolumn{7}{|c|}{ Hemagglutinin inhibition (HI) antibody titer with the antiserum of different viruses ${ }^{\text {a) }}$} \\
\hline & $\mathrm{CK} / \mathrm{SX} / 2 / 06$ & $\mathrm{CK} / \mathrm{JS} / 18 / 08$ & $\mathrm{CK} / \mathrm{HeB} / \mathrm{A} / 12$ & $\mathrm{CK} / \mathrm{SD} / \mathrm{A}-8 / 11$ & $\mathrm{CK} / \mathrm{LN} / \mathrm{S} 4068 / 11$ & $\mathrm{CK} / \mathrm{HeB} / 3 / 13$ & $\mathrm{CK} / \mathrm{GS} / 6 / 12$ \\
\hline $\mathrm{CK} / \mathrm{SX} / 2 / 06(\mathrm{H} 5 \mathrm{~N} 1)$ & $128^{\mathrm{b})}$ & 8 & 4 & 2 & 2 & 2 & 2 \\
\hline $\mathrm{CK} / \mathrm{JS} / 18 / 08$ (H5N1) & 2 & 256 & 4 & 128 & 2 & 4 & 2 \\
\hline $\mathrm{CK} / \mathrm{HeB} / \mathrm{A} / 12$ (H5N2) & 4 & 4 & 128 & 4 & 16 & 16 & 16 \\
\hline CK/SD/A-8/11 (H5N1) & 4 & 64 & 8 & 128 & 4 & 8 & 4 \\
\hline CK/LN/S4068/11 (H5N1) & 4 & 4 & 64 & 4 & 64 & 32 & 32 \\
\hline $\mathrm{CK} / \mathrm{HeB} / 3 / 13$ (H5N2) & 8 & 8 & 64 & 8 & 64 & 512 & 64 \\
\hline CK/GS/6/12 (H5N1) & 8 & 8 & 64 & 8 & 32 & 32 & 128 \\
\hline CK/LN/S4092/11 (H5N1) & 8 & 8 & 64 & 8 & 64 & 64 & 64 \\
\hline $\mathrm{CK} / \mathrm{NX} / 2 / 12(\mathrm{H} 5 \mathrm{~N} 1)$ & 4 & 8 & 64 & 4 & 32 & 32 & 32 \\
\hline
\end{tabular}

a) Antisera were generated by vaccinating specific-pathogen-free chickens with hemagglutinin-expressing plasmids of the indicated viruses. b) Homologous HI titers are shown in boldface. 


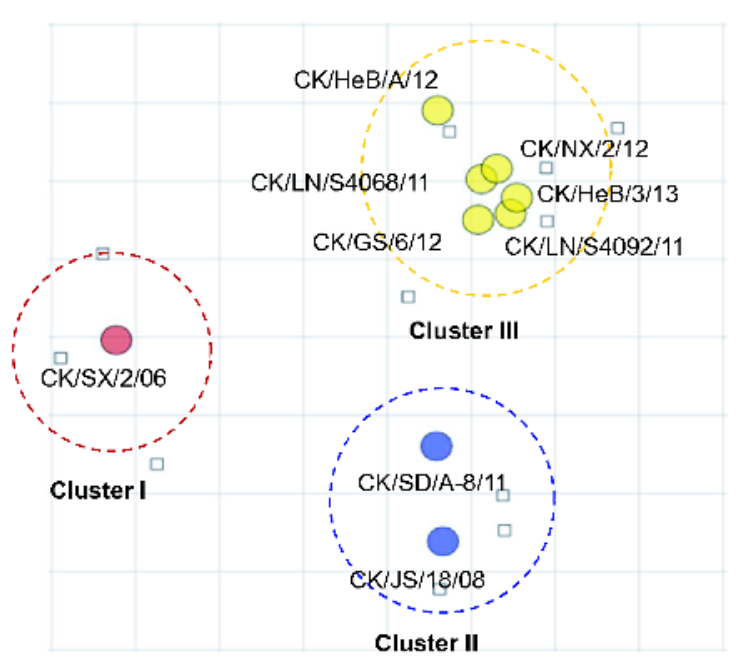

Figure 1 Antigenic cartography of clade $7.2 \mathrm{H} 5$ viruses. Open squares and filled circles represent the positions of the antisera and viruses, respectively. The spacing of one gridline corresponds to an $\mathrm{HI}$ measurement, which equals a 2 -fold difference in the HI assay. Both axes represent antigenic distance.

reactivity of the virus with the monoclonal antibody (MAb) that targets that epitope, and further alter the antigenicity of the influenza virus. To investigate the reactivity of CK/JS/18/ 08 and $\mathrm{CK} / \mathrm{HeB} / \mathrm{A} / 12$ with MAbs targeted to $\mathrm{CK} / \mathrm{SX} / 2 / 06$, we successfully generated eight MAbs, named SX-53, SX59, SX-88, SX-89, SX-25, SX-16, SX-64, and SX-84, against the HA protein of $\mathrm{CK} / \mathrm{SX} / 2 / 06$ as described in the Materials and Methods section. HI tests showed that MAbs SX-64 and SX-84 reacted well with these three viruses; the other six MAbs (SX-16, SX-25, SX-53, SX-59, SX-88, and SX-89) only reacted with CK/SX/2/06 and not with CK/JS/ $18 / 08$ and $\mathrm{CK} / \mathrm{HeB} / \mathrm{A} / 12$ (Figure 2). To eliminate the impact of other virus proteins on the $\mathrm{HI}$ assay, we generated three reassortants bearing the HA gene from the three representative viruses, the NA gene of $\mathrm{CK} / \mathrm{SX} / 2 / 06$, and the six internal genes of A/Puerto Rico/8/1934 (H1N1) (PR8) by using reverse genetics. Of note, we also removed the multiple basic amino acids in the cleavage site of the HA gene of these reassortants to eliminate their potential virulence to poultry and mammals. The rescued viruses were named PR8SX/HA, PR8-JS/HA, and PR8-HeB/HA, and the HI test results indicated that the three reassortants and their HA gene donors had similar reaction patterns to the eight MAbs (Figure 2). These results show that the epitopes targeted by MAbs SX-64 and SX-84 are conserved in all three viruses, but the epitopes targeted by the other six MAbs have changed in $\mathrm{CK} / \mathrm{JS} / 18 / 08$ and $\mathrm{CK} / \mathrm{HeB} / \mathrm{A} / 12$.

\section{The E131N mutation and a leucine insertion at position 134 in $\mathrm{HA}$ change the reactivity of PR8-SX/HA with different MAbs}

A detailed amino acid comparison revealed that there were

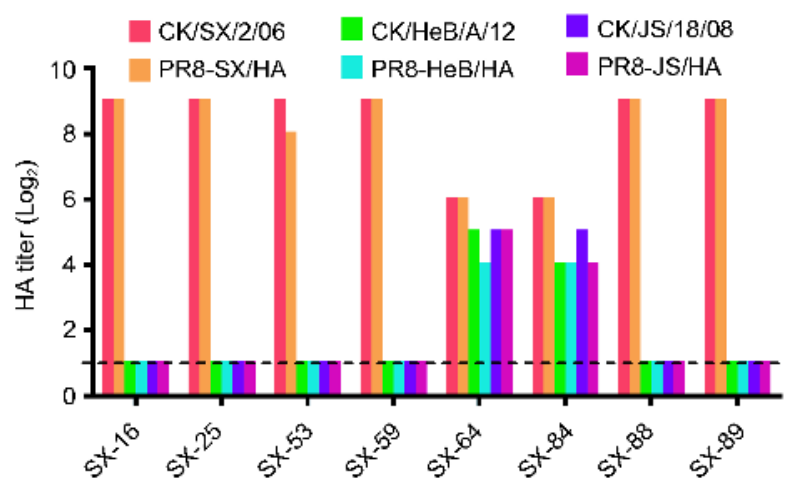

Figure 2 Cross-reactivity of different clade 7.2 viruses with monoclonal antibodies (MAbs) as determined by the HI test. MAbs were generated against the $\mathrm{HA}$ of the index virus $\mathrm{CK} / \mathrm{SX} / 2 / 06$; the dashed line indicates the limit of detection.

18 amino acid differences between CK/SX/2/06 and CK/JS/ 18/08 and 30 amino acid differences between CK/SX/2/06 and $\mathrm{CK} / \mathrm{HeB} / \mathrm{A} / 12$ in their $\mathrm{HA} 1$ protein (Figure $3 \mathrm{~A}$ ). Seventeen of these amino acids (shown in red in Figure $3 \mathrm{~A}$ ) are located on the surface of the globular head of the HA protein, as shown in the simulated 3D structure of $\mathrm{CK} / \mathrm{SX} / 2 / 06 \mathrm{HA}$ (Figure 3B) generated by using SWISS-MODEL (www. swissmodel.expasy.org)(Waterhouse et al., 2018) and Pymol software.

To pinpoint which of these amino acids contribute to the different reactivity to the MAbs, we generated 15 mutants by reverse genetics in the PR8-SX/HA background; each of these mutants contains one or two of the seventeen amino acids showed in red in Figure 3A that appeared in the HA1 of $\mathrm{CK} / \mathrm{JS} / 18 / 08$ and/or $\mathrm{CK} / \mathrm{HeB} / \mathrm{A} / 12$. These mutants were designated SX/HA-K62E，SX/HA-E78K，SX/HA-A93V, SX/HA-H117R, SX/HA-K122E，SX/HA-S126F，SX/HAS126H，SX/HA-E131N-A132T, SX/HA-134L ${ }^{\text {ins }}$ (leucine insertion at position 134), SX/HA-G143E-K144N, SX/HAK144D， SX/HA-T171A-E174K， SX/HA-D187N-T189A, SX/HA-V192I-K193M, and SX/HA-V192K-K193M (H3 numbering throughout).

We then performed HI tests with the 15 mutants and the six MAbs (SX-16, SX-25, SX-53, SX-59, SX-88, and SX-89) that reacted with $\mathrm{CK} / \mathrm{SX} / 2 / 06$ but not with $\mathrm{CK} / \mathrm{JS} / 18 / 08$ and $\mathrm{CK} / \mathrm{HeB} / \mathrm{A} / 12$ (Figure 2). We found that the mutant SX/HAE131N-A132T did not react with any of the MAbs, and that the mutant SX/HA-134L ${ }^{\text {ins }}$ did not react with the four of the MAbs but reacted with MAbs SX-88 and SX-89. The other 13 mutants reacted with all six of the MAbs. These findings demonstrate that MAbs SX-16, SX-25, SX-53, and SX-59 have similar reaction patterns with the mutants; they reacted with 13 of the 15 mutants, but not with the mutant SX/HAE131N-A132T or the mutant SX/HA-134L ${ }^{\text {ins }}$. The MAbs SX-88 and SX-89 have similar reaction patterns with the mutants; they reacted with 14 of the 15 mutants, but not with 

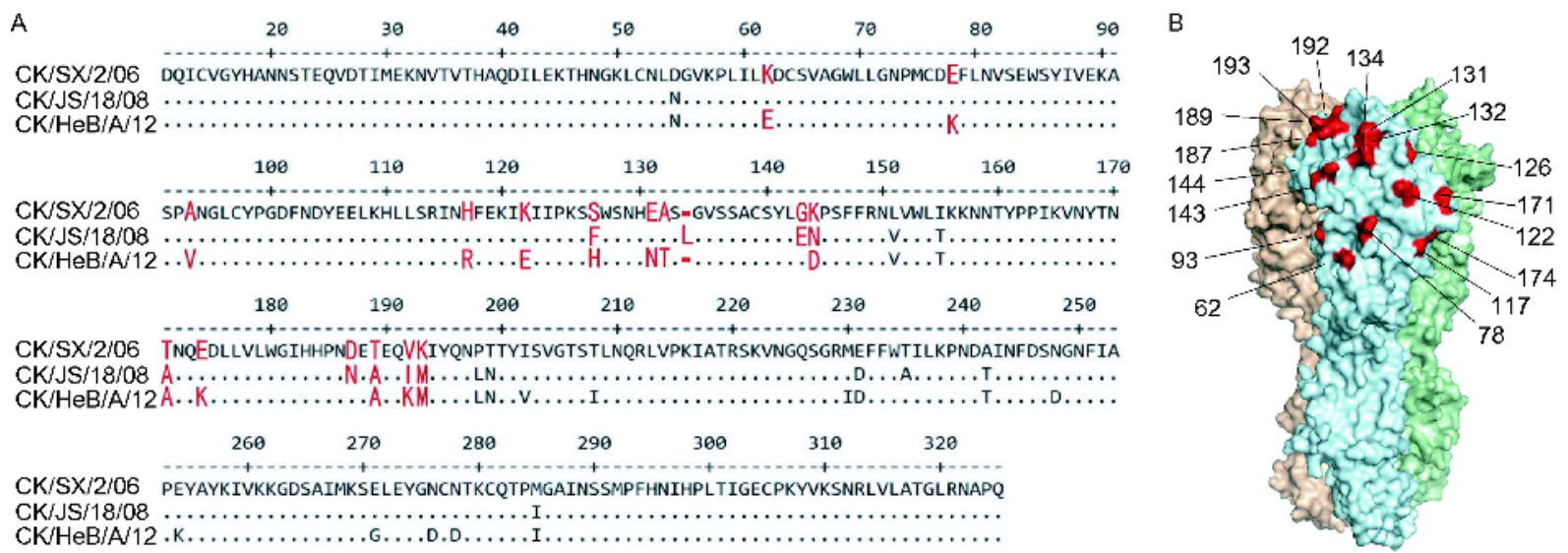

Figure 3 Amino acid differences in the HA1 of three representative clade 7.2 viruses. A, Amino acid sequence alignment (generated with MegAlign software) of the HA1 polypeptide of CK/SX/2/06, CK/JS/18/08, and CK/HeB/A/12. The dots show the amino acid residues that are the same as those of CK/ $\mathrm{SX} / 2 / 06$. The dash indicates the lack of a residue compared with CK/JS/18/08 (amino acid numbering is based on H3 HA). The key amino acids in the head of the HA1 trimer that are different in the three viruses are highlighted in red. B, 3D structure of the HA protein obtained by using SWISS-MODEL; the image was drawn with Pymol software. The numbers show the positions of the key amino acids in the head of the HA1 trimer that are different in the three viruses.

the mutant SX/HA-E131N-A132T, indicating that the four MAbs SX-16, SX-25, SX-53, and SX-59 may recognize the same epitope, whereas MAbs SX-88 and SX-89 recognize another epitope (Figure 4). Therefore, we selected two MAbs, SX-53 and SX-88, for further study.

The mutant containing double mutations at positions 131 and 132 of HA (SX/HA-E131N-A132T) did not react with any of the MAbs tested. Therefore, to investigate which of these two mutations plays a role in the lack of reactivity, we generated two more mutants, SX/HA-E131N and SX/HAA132T, in the PR8-SX/HA background and tested their reactivity with MAbs SX-53 and SX-88. We found that the mutant SX/HA-E131N did not react with either of the two MAbs, whereas mutant SX/HA-A132T reacted with both MAbs (Figure 5A), indicating that the E131N mutation eliminated the reactivity of PR8-SX/HA with the two MAbs.

\section{The N131E mutation and the $134 \mathrm{~L}$ deletion alter the reactivity of PR8-HeB/HA and PR8-JS/HA with MAbs SX-53 and SX-88}

Since the E131N mutation and the 134L insertion were detected in the $\mathrm{CK} / \mathrm{HeB} / \mathrm{A} / 12$ virus and the $\mathrm{CK} / \mathrm{JS} / 18 / 08$ virus, respectively, we investigated how these mutations would affect the reactivity of the $\mathrm{CK} / \mathrm{HeB} / \mathrm{A} / 12$ virus and the $\mathrm{CK} /$ $\mathrm{JS} / 18 / 08$ virus with MAbs SX-53 and SX-88. We generated two mutants by introducing the HA N131E into the PR8$\mathrm{HeB} / \mathrm{HA}$ virus and deleting the HA $134 \mathrm{~L}$ of the PR8-JS/HA virus, and designated the two mutants HeB/HA-N131E and JS/HA-134L ${ }^{\text {del }}$, respectively. HI test results showed that the parent viruses PR8-HeB/HA and PR8-JS/HA did not react with MAbs SX-53 and SX-88, that the mutant HeB/HAN131E reacted with MAb SX-88 but not with MAb SX-53, and that the mutant JS/HA-134L ${ }^{\text {del }}$ reacted with MAbs SX-
53 and SX-88 (Figure 5A). Of note, the 134L insertion in the HA also appeared in the other virus in the antigenic cluster II, and the $131 \mathrm{~N}$ mutation was present in all of the other five viruses in the antigenic cluster III shown in Figure 1.

\section{The E131N mutation in HA forms a new N-linked gly- cosylation site -NAS- at positions 131-133}

The E131N substitution in HA formed a new N-linked glycosylation site. We performed Western blotting analysis of the HA polypeptides of PR8-SX/HA, SX/HA-E131N, PR8$\mathrm{HeB} / \mathrm{HA}$, and $\mathrm{HeB} / \mathrm{HA}-\mathrm{N} 131 \mathrm{E}$ to investigate whether this potential glycosylation site was glycosylated. The HA of SX/ HA-E131N showed decreased mobility compared with that of PR8-SX/HA, whereas HeB/HA-N131E HA showed increased mobility compared with that of PR8-HeB/HA because of the single amino acid mutation N131E (Figure 5B). These results confirm that -NXS- at positions 131-133 in the HA proteins of $\mathrm{CK} / \mathrm{HeB} / \mathrm{A} / 12$ and SX/HA-E131N was indeed glycosylated.

\section{DISCUSSION}

Antigenic drift frequently occurs during influenza virus circulation in nature, and an antigenically variable virus that escapes from immunity induced by prior infection or vaccination will cause a human influenza epidemic or an avian influenza outbreak. We used the clade $7.2 \mathrm{H} 5$ viruses as models to explore the molecular determinants of the antigenic drift of influenza viruses, and found that the clade 7.2 viruses isolated between 2006 and 2013 formed three distinct antigenic groups. We generated and identified two key MAbs SX-53 and SX-88 that reacted with the index strain CK/SX/ 

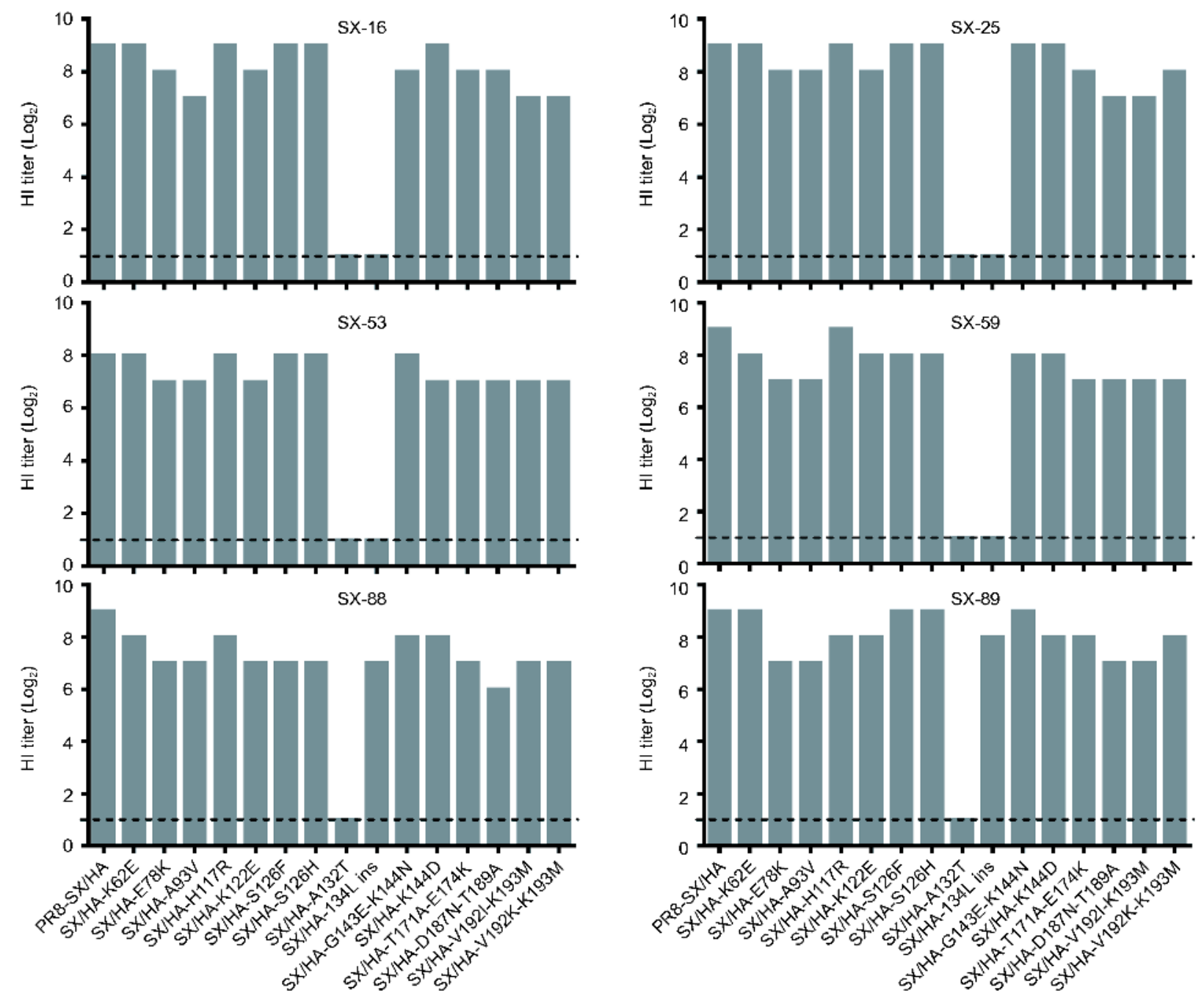

Figure 4 The reactivity of different HA mutants of PR8-SX/HA virus with six selected MAbs. The reactivity was tested by using the HI assay; the dashed lines indicate the limit of detection.

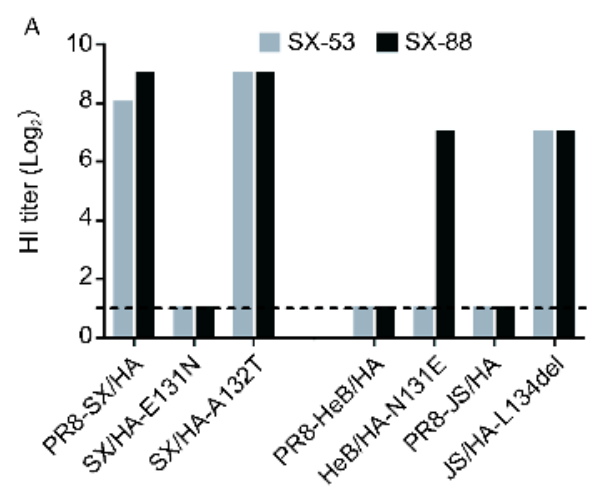

B

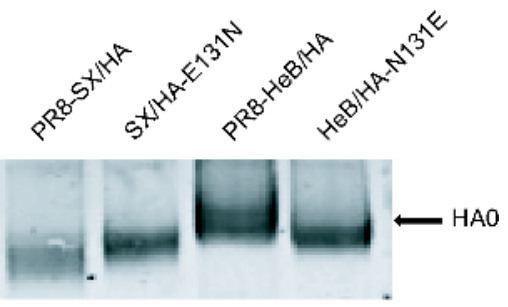

Figure 5 The reactivity of HA mutant viruses with different virus backbones with the MAbs SX-53 and SX-88. A, The reactivity was tested by using the HI assay; the dashed lines indicate the limit of detection. B, Mobility analysis of HA proteins. Lysates of viruses were analyzed by use of SDS-polyacrylamide gel electrophoresis (SDS-PAGE) and Western blot analysis. Chicken anti-H5 antiserum was used as the primary antibody. Protein bands were detected by using the Odyssey infrared imaging system after incubation with IRDyeTM700DX-conjugated secondary antibody. 
$2 / 06$ virus but not with representative viruses from the other two antigenic groups in the HI assay. We then constructed a series of HA mutants of clade 7.2 H5 viruses and tested their reactivity with the two MAbs, and found that glycosylation at positions 131-133 in HA and a single amino acid insertion of $134 \mathrm{~L}$ in HA independently affected the reactivity of the clade 7.2 viruses with the two MAbs.

The glycosylation of HA affects the properties of influenza viruses in multiple ways (Gao et al., 2009; Kawaoka et al., 1984; Ping et al., 2008; Skehel et al., 1984; Zhao et al., 2017). Gao et al. previously reported that the loss of glycosylation at positions 158-160 of HA was responsible for the binding to $\alpha 2,6$-sialylated glycans of the $\mathrm{H} 5 \mathrm{~N} 1$ viruses $\mathrm{A} /$ duck/Guangxi/35/2001 and A/Bar-headed goose/3/2005 and was critical for the transmission of these viruses in guinea pigs (Gao et al., 2009). Zhao et al. reported that loss of glycosylation at positions $158-160$ of HA attenuated the H5N1 virus A/chicken/Vietnam-Ca Mau/1180/2006 in mice, but did not alter its receptor binding property (Zhao et al., 2017). In the present study, neither PR8-SX/HA nor HeB/ HA-N132E had a glycosylation site at positions 131-133 of HA. PR8-SX/HA reacted with both MAb SX-53 and MAb SX-88, whereas HeB/HA-N132E reacted with MAb SX-88 but not MAb SX-53 (Figure 5A). These findings suggest that HA glycosylation at the same position in different viruses may function differently, possibly because of differences in other regions of the protein.

Deletions and insertions regularly occur in the genome of influenza viruses, and such genome alterations always cause changes in the biological properties of the viruses. Previous studies have shown that the deletion of amino acids 191 to 195 of the NS1 protein is critical for the attenuation of the swine H5N1 virus A/swine/Fujian/1/2003 in chickens, mainly because this deletion impairs NS1 dimerization, thereby reducing the interaction of NS1 with TRIM25 and double-stranded RNAs (Wang et al., 2018; Zhu et al., 2008). An insertion of amino acids in the HA cleavage site that creates the motif -BXBR- $(\mathrm{B}=$ basic amino acids arginine or lysine, $\mathrm{X}=$ any amino acid, $\mathrm{R}=$ arginine) or $-\mathrm{RXXR}$ - usually transforms low pathogenic $\mathrm{H} 5$ or $\mathrm{H} 7$ viruses into their highly pathogenic forms (Neumann and Kawaoka, 2006; Senne et al., 1996; Shi et al., 2017). In this study, we found that the $134 \mathrm{~L}$ insertion in the HA of $\mathrm{CK} / \mathrm{SX} / 02 / 06$ only prevented the reactivity of the virus with the SX-53-like MAbs (Figure 4). In contrast, the $134 \mathrm{~L}$ insertion in the HA of CK/JS/18/08 prevented the reactivity of the virus with all of the SX-53like and SX-88-like MAbs (Figure 2), suggesting that other amino acid changes around this area in the HA of the two viruses may contribute to the different interactions of the two viruses with the MAb SX-88-like MAbs.

In summary, here we provided experimental evidence that glycosylation and an amino acid insertion or deletion in HA alter the interaction of $\mathrm{H} 5 \mathrm{~N} 1$ viruses with MAbs, and therefore have a role in the antigenic variation of influenza viruses. These findings are important for the screening of field antigenic variants. Moreover, these data highlight the importance of evaluating the antigenic properties of viruses that have changes to their glycosylation sites and/or amino acid insertions or deletions in their HA.

\section{MATERIALS AND METHODS}

\section{Ethics statements and facility}

All animal experiments were conducted in biosecurity level 2 facilities approved by the Harbin Veterinary Research Institute (HVRI) of the Chinese Academy of Agricultural Sciences (CAAS). This study was carried out in strict accordance with the recommendations in the Guide for the Care and Use of Laboratory Animals of the Ministry of Science and Technology of the People's Republic of China.

\section{Viruses and cells}

The wild-type clade 7.2 H5N1 avian influenza viruses in this study were isolated from domestic poultry and were reported previously (Li et al., 2010; Liu et al., 2016). The 293T and MDCK cells were cultured in Dulbecco's modified Eagle's medium supplemented with $10 \%$ fetal calf serum and were incubated at $37^{\circ} \mathrm{C}$ in $5 \% \mathrm{CO}_{2}$. Sp2/0 cells were cultured in RPMI 1640 medium with $10 \%$ fetal calf serum and incubated at $37^{\circ} \mathrm{C}$ in $5 \% \mathrm{CO}_{2}$.

\section{Antisera}

The antisera specifically against the HA protein were prepared by the method described by Jiang et al. (Jiang et al., 2007). Briefly, the HA genes of clade 7.2 viruses were cloned into the plasmid vector pCAGGS. The plasmids were diluted to $1 \mu \mathrm{g} \mu \mathrm{L}^{-1}$ and then injected in a $100-\mu \mathrm{L}$ volume at the leg muscle of 4-week-old specific-pathogen-free (SPF) chickens. Antisera were collected 3 weeks post-vaccination.

\section{Antigenic cartography}

Analysis of antigenic properties was performed using the antigenic cartography methods described previously (Smith et al., 2004). The antigen cartography of the viruses was constructed by using Antigenic Cartography software (http:// www.antigenic-cartography.org/).

\section{Monoclonal antibodies}

The MAbs used in this study were prepared by a modification of the method described by Köhler and Milstein (Köhler 
and Milstein, 1976). Briefly, BALB/c mice were injected intramuscularly with three doses of $50 \mu \mathrm{g}\left(1 \mu \mathrm{g} \mu \mathrm{L}^{-1}\right)$ of plasmid pCAGGS-SX-HA, which expresses the HA gene of the $\mathrm{CK} / \mathrm{SX} / 2 / 06$ virus, at 6 weeks old, 9 weeks old, and 11 weeks old. Three days after the third dose, the mice were sacrificed and their spleens were removed and disrupted to obtain splenocytes. $\mathrm{Sp} 2 / 0$ cells and spleen cells were fused with polyethylene glycol. The fused hybridoma cells were cultured in a 96-well plate. Twenty-four hours later, half the medium was replaced with HAT medium; this was repeated every day for one week. The positive hybridoma cells were screened by using the HI assay. Positive hybridoma cells were cloned by limiting dilution and seeded into 96-well plates at 1 cell per $100 \mu \mathrm{L}$ per well. After 3 rounds of cloning, single positive clones were propagated for largescale culture. The cloned hybridoma cells were intraperitoneally injected into BALB/c mice. Ascites fluid was collected 7 days later.

\section{HI test}

The HI test was performed following standard methods. Briefly, $25 \mu \mathrm{L}$ of 2-fold serially diluted antisera or MAbs was mixed with $25 \mu \mathrm{L}$ of $4 \mathrm{HA}$ units of virus, and incubated for $30 \mathrm{~min}$ at room temperature. Subsequently, $50 \mu \mathrm{L}$ of $0.5 \%$ chicken red blood cells was added to each well and incubated for $40 \mathrm{~min}$ at room temperature. The $\mathrm{HI}$ titer is the highest dilution of serum or monoclonal antibody that completely inhibits hemagglutination.

\section{Virus rescue}

Reassortant viruses containing HA with the modified cleavage site of different clade 7.2 viruses and the NA gene of $\mathrm{CK} / \mathrm{SX} / 2 / 06$ in the background of $\mathrm{A} / \mathrm{PR} / 8 / 34$ were generated by reverse genetics as described previously (Li et al., 2005). Mutations were introduced into the HA gene by PCR using the QuickChange Site-Directed Mutagenesis kit. Primer sequences are available upon request. The rescued viruses were fully sequenced to ensure the absence of unwanted mutations.

\section{Western blot}

Viruses were propagated in the allantoic fluid of embryonated SPF chicken eggs and purified from the allantoic fluid by centrifugation. The concentrated viruses were re-suspended with PBS and inactivated with $\beta$-propionolactone. The samples were analyzed by SDS-PAGE and Western blotting as described previously (Ping et al., 2008). The chicken antisera induced by the pCAGGS-HA were used as the primary antibody, and IRDyeTM700DX-conjugated rabbit anti-chicken antiserum was used as the secondary antibody (Sigma).

Compliance and ethics The authors declare that they have no conflict of interest.

Acknowledgements This work was supported by the National Natural Science Foundation of China (31521005, 31672593), the National Key $R \& D$ Program of China (2016YFD0500201, 2016YFD0500203), the China Agriculture Research System (CARS-41-G12), and by the Japan Initiative for Global Research Network on Infectious Diseases from the Ministry of Education, Culture, Sports, Science, and Technology (MEXT) of Japan.

\section{References}

Fouchier, R., Schneeberger, P., Rozendaal, F., Broekman, J., Kemink, S., Munster, V., Kuiken, T., Rimmelzwaan, G., Schutten, M., Van Doornum, G., et al. (2004). Avian influenza A virus (H7N7) associated with human conjunctivitis and a fatal case of acute respiratory distress syndrome. Proc Natl Acad Sci USA 101, 13561361.

Gao, Y., Zhang, Y., Shinya, K., Deng, G., Jiang, Y., Li, Z., Guan, Y., Tian, G., Li, Y., Shi, J., et al. (2009). Identification of amino acids in HA and $\mathrm{PB} 2$ critical for the transmission of $\mathrm{H} 5 \mathrm{~N} 1$ avian influenza viruses in a mammalian host. PLoS Pathog 5, e1000709.

Herfst, S., Schrauwen, E., Linster, M., Chutinimitkul, S., de Wit, E., Munster, V., Sorrell, E., Bestebroer, T., Burke, D., Smith, D., et al. (2012). Airborne transmission of influenza A/H5N1 virus between ferrets. Science 336, 1534-1541.

Imai, M., Watanabe, T., Hatta, M., Das, S., Ozawa, M., Shinya, K., Zhong, G., Hanson, A., Katsura, H., Watanabe, S., et al. (2012). Experimental adaptation of an influenza H5 HA confers respiratory droplet transmission to a reassortant $\mathrm{H} 5 \mathrm{HA} / \mathrm{H} 1 \mathrm{~N} 1$ virus in ferrets. Nature 486, 420-428.

Jiang, Y., Yu, K., Zhang, H., Zhang, P., Li, C., Tian, G., Li, Y., Wang, X., Ge, J., Bu, Z., et al. (2007). Enhanced protective efficacy of H5 subtype avian influenza DNA vaccine with codon optimized HA gene in a pCAGGS plasmid vector. Antiviral Res 75, 234-241.

Kawaoka, Y., Naeve, C., and Webster, R. (1984). Is virulence of H5N2 influenza viruses in chickens associated with loss of carbohydrate from the hemagglutinin? Virology 139, 303-316.

Kimble, J., Sorrell, E., Shao, H., Martin, P., and Perez, D. (2011). Compatibility of H9N2 avian influenza surface genes and 2009 pandemic H1N1 internal genes for transmission in the ferret model. Proc Natl Acad Sci USA 108, 12084-12088.

Köhler, G., and Milstein, C. (1976). Derivation of specific antibodyproducing tissue culture and tumor lines by cell fusion. Eur J Immunol $6,511-519$.

Li, C., Bu, Z., and Chen, H. (2014a). Avian influenza vaccines against H5N1 'bird flu'. Trends Biotech 32, 147-156.

Li, X., Shi, J., Guo, J., Deng, G., Zhang, Q., Wang, J., He, X., Wang, K., Chen, J., Li, Y., et al. (2014b). Genetics, receptor binding property, and transmissibility in mammals of naturally isolated H9N2 Avian Influenza viruses. PLoS Pathog 10, e1004508.

Li, Y., Shi, J., Zhong, G., Deng, G., Tian, G., Ge, J., Zeng, X., Song, J., Zhao, D., Liu, L., et al. (2010). Continued evolution of H5N1 influenza viruses in wild birds, domestic poultry, and humans in China from 2004 to 2009. J Virol 84, 8389-8397.

Li, Z., Chen, H., Jiao, P., Deng, G., Tian, G., Li, Y., Hoffmann, E., Webster, R.G., Matsuoka, Y., and Yu, K. (2005). Molecular basis of replication of duck H5N1 influenza viruses in a mammalian mouse model. J Virol 79, 12058-12064.

Liu, L., Zeng, X., Chen, P., Deng, G., Li, Y., Shi, J., Gu, C., Kong, H., Suzuki, Y., Jiang, Y., et al. (2016). Characterization of clade 7.2 H5 avian influenza viruses that continue to circulate in chickens in China. J 
Virol 90, 9797-9805.

Neumann, G., and Kawaoka, Y. (2006). Host range restriction and pathogenicity in the context of influenza pandemic. Emerg Infect Dis $12,881-886$.

Ping, J., Li, C., Deng, G., Jiang, Y., Tian, G., Zhang, S., Bu, Z., and Chen, H. (2008). Single-amino-acid mutation in the HA alters the recognition of H9N2 influenza virus by a monoclonal antibody. Biochem BioPhys Res Commun 371, 168-171.

Senne, D., Panigrahy, B., Kawaoka, Y., Pearson, J., Suss, J., Lipkind, M., Kida, H., and Webster, R. (1996). Survey of the hemagglutinin (HA) cleavage site sequence of $\mathrm{H} 5$ and $\mathrm{H} 7$ avian influenza viruses: amino acid sequence at the HA cleavage site as a marker of pathogenicity potential. Avian Dis 40, 425-437.

Shi, J., Deng, G., Kong, H., Gu, C., Ma, S., Yin, X., Zeng, X., Cui, P., Chen, Y., Yang, H., et al. (2017). H7N9 virulent mutants detected in chickens in China pose an increased threat to humans. Cell Res 27, 1409-1421.

Shi, J., Deng, G., Ma, S., Zeng, X., Yin, X., Li, M., Zhang, B., Cui, P., Chen, Y., Yang, H., et al. (2018). Rapid evolution of H7N9 highly pathogenic viruses that emerged in China in 2017. Cell Host Microbe 24, 558-568.e7.

Skehel, J., Stevens, D., Daniels, R., Douglas, A., Knossow, M., Wilson, I., and Wiley, D. (1984). A carbohydrate side chain on hemagglutinins of Hong Kong influenza viruses inhibits recognition by a monoclonal antibody.. Proc Natl Acad Sci USA 81, 1779-1783.

Smith, D., Lapedes, A., de Jong, J., Bestebroer, T., Rimmelzwaan, G., Osterhaus, A., and Fouchier, R. (2004). Mapping the antigenic and genetic evolution of influenza virus. Science 305, 371-376.

Sorrell, E., Wan, H., Araya, Y., Song, H., and Perez, D. (2009). Minimal molecular constraints for respiratory droplet transmission of an avianhuman H9N2 influenza A virus. Proc Natl Acad Sci USA 106, 75657570.

Swayne, D. (2012). Impact of vaccines and vaccination on global control of avian influenza. Avian Dis 56, 818-828.

Wang, J., Zeng, Y., Xu, S., Yang, J., Wang, W., Zhong, B., Ge, J., Yin, L., $\mathrm{Bu}, \mathrm{Z}$., Shu, H., et al. (2018). A naturally occurring deletion in the effector domain of $\mathrm{H} 5 \mathrm{~N} 1$ swine influenza virus nonstructural protein 1 regulates viral fitness and host innate immunity. J Virol 92, pii: e0014918.

Waterhouse, A., Bertoni, M., Bienert, S., Studer, G., Tauriello, G.,
Gumienny, R., Heer, F., de Beer, T., Rempfer, C., Bordoli, L., et al. (2018). SWISS-MODEL: homology modelling of protein structures and complexes. Nucleic Acids Res 46, W296-W303.

Wei, X., and Cui, J. (2018). Why were so few people infected with H7N9 influenza A viruses in China from late 2017 to 2018? Sci China Life Sci $61,1442-1444$.

WHO-OIE-FAO H5 evolution working group. (2008). Toward a unified nomenclature system for highly pathogenic avian influenza virus (H5N1). Emerg Infect Dis 14, e1.

World Health Organization. (2014). Influenza virus infections in humans (February 2014). http://www.who.int/influenza/human_animal_interface/virology_laboratories_and_vaccines/influenza_virus_infections_humans_feb14.pdf?ua $=1$.

World Health Organization. (2018a). Influenza at the human-animal interface. http://www.who.int/influenza/human_animal_interface/Influenza_Summary_IRA_HA_interface_02_03_2018.pdf?ua=2011.

World Health Organization. (2018b). Cumulative number of confirmed human cases of avian influenza $\mathrm{A}(\mathrm{H} 5 \mathrm{~N} 1)$ reported to WHO. http:// www.who.int/influenza/human_animal_interface/H5N1_cumulative_table_archives/en/.

Zeng, X., Tian, G., Shi, J., Deng, G., Li, C., and Chen, H. (2018). Vaccination of poultry successfully eliminated human infection with H7N9 virus in China. Sci China Life Sci 61, doi: https://doi.org/ 10.1007/s11427-018-9420-1.

Zhang, Q., Shi, J., Deng, G., Guo, J., Zeng, X., He, X., Kong, H., Gu, C., Li, X., Liu, J., et al. (2013a). H7N9 influenza viruses are transmissible in ferrets by respiratory droplet. Science 341, 410-414.

Zhang, Y., Zhang, Q., Kong, H., Jiang, Y., Gao, Y., Deng, G., Shi, J., Tian, G., Liu, L., Liu, J., et al. (2013b). H5N1 hybrid viruses bearing 2009/ H1N1 virus genes transmit in guinea pigs by respiratory droplet. Science 340, 1459-1463.

Zhao, D., Liang, L., Wang, S., Nakao, T., Li, Y., Liu, L., Guan, Y., Fukuyama, S., Bu, Z., Kawaoka, Y., et al. (2017). Glycosylation of the hemagglutinin protein of $\mathrm{H} 5 \mathrm{~N} 1$ influenza virus increases its virulence in mice by exacerbating the host immune response. J Virol 91, pii: e0221516.

Zhu, Q., Yang, H., Chen, W., Cao, W., Zhong, G., Jiao, P., Deng, G., Yu, K., Yang, C., Bu, Z., et al. (2008). A naturally occurring deletion in its NS gene contributes to the attenuation of an $\mathrm{H} 5 \mathrm{~N} 1$ swine influenza virus in chickens. J Virol 82, 220-228. 